\title{
Pharmacoeconomic Modeling of Prior-Authorization Intervention for COX-2 Specific Inhibitors in a 3-Tier Copay Plan
}

\author{
JANE STACY, PharmD; ELIZABETH SHAW, MSIE; MICHELE D. ARLEDGE, PharmD; and DONNA HOWELL-SMITH, RPh, MBA
}

\begin{abstract}
OBJECTIVE: To determine from a health plan perspective the cost-effectiveness of cyclooxygenase-2 (COX-2) specific inhibitors, with and without a prior-authorization $(\mathrm{PA})$ process.

METHODS: A modeling exercise was employed, based on prescription drug claims for a managed care organization with 3.8 million health maintenance organization (HM0) and preferred provider organization (PP0)

members. Drug claims revealed 96,154 members $(2.9 \%$ of the 3.3 million members with a pharmacy benefit) who received either one or more prescriptions for a COX-2 drug or a nonspecific nonsteroidal anti-inflammatory drug (NSAID). These patients were stratified into 2 groups for further analysis, those having a concurrent proton pump inhibitor (PPI) and those without a concurrent PPI. Decision analysis was used to estimate the cost-effectiveness of COX-2 therapy. Actual health plan drug claims data were used to determine utilization and prescribing patterns of nonspecific NSAIDs, COX-2 specific inhibitors, and PPIs. Results from the literature from 8 clinical trials were employed to determine the probability of a serious gastrointestinal (GI) event. Cost-effectiveness analysis (CEA) was used to determine the cost of each therapy, including the predicted cost to treat a serious $\mathrm{Gl}$ event in a drug benefit design with PA versus a benefit design without PA.
\end{abstract}

RESULTS: Cost-effectiveness analysis (CEA) showed that the cost per success (no serious Gl event) for Cox-2 specific inhibitors with PA was \$278 versus \$422 without PA.

CONCLUSIONS: The one-year model predicted that costs associated with an increase in COX-2 utilization after removal of PA would exceed the costs to administer PA and treat NSAID-related serious GI events in the managed care population. Based upon this CEA, PA appears to be an effective tool to manage COX-2 pharmacy costs. Further examination of the medical claims would be useful to validate the assumed Gl event rates with or without PA and to further demonstrate more definitively the value of a PA program for COX-2 drugs.

KEYWORDS: Nonsteroidal anti-inflammatory drugs, COX-2 selective inhibitors, Prior authorization, Managed care, Serious GI events

J Managed Care Pharm. 2003;9(4):327-34
1 rior authorization (PA) is a common tool within the managed care environment. Demand has increased for managed care plans to provide access to new and more costly drugs for their members. Many managed care organizations (MCOs) use the PA process as a way to control cost by assuring that members have tried less-expensive alternatives before meeting the criteria for use of the requested agent. With direct-to-consumer (DTC) advertising a mainstay in the current health care environment, some prescribers feel consumers have placed increased demands on physicians to prescribe advertised products. $^{1,2}$ Health plans are continually looking for ways to control pharmacy cost trend through benefit design. To maintain optimal access to medications, it will become increasingly important to be cognizant of the effects of prescription drugs on total medical outcomes, including costs outcomes and the actuarial-underwriting process.

Benefit (re)design is likely the best way to address not only cost and access but also their impact on total medical outcomes. PA has historically been one benefit design feature frequently used by prescription benefit managers (PBMs) and health plans in the United States to control the use of higher-cost drugs. ${ }^{3}$ Today, one of these high-cost drivers is the cyclooxygenase 2 (COX-2) specific inhibitor class of drugs. COX-2 specific inhibitors cost an average of $\$ 80$ more per month than their nonspecific nonsteroidal anti-inflammatory alternatives. For example, the average ingredient cost of a 1-month supply of a COX-2 specific inhibitor is $\$ 100$, while the average ingredient cost of a generic nonspecific NSAID such as naproxen is $\$ 20$ per month.

COX-2 specific inhibitors are indicated for treating osteoarthritis, rheumatoid arthritis, and acute pain.4-6 These medications may be alternatives for plan members who are intolerant to nonspecific NSAIDs or who have a high risk of developing gastrointestinal (GI) adverse events, particularly ulcers or GI bleeding. ${ }^{7-9}$ The main disadvantage of treatment with NSAIDs is the adverse effects that can be associated with their use, including GI discomfort and more serious events such as bleeding and ulceration. ${ }^{10-13}$ For serious GI events (defined as bleeding, ulceration, or perforation requiring hospitalization and/or additional treatment) the incidence is 2 to 4 times greater for those members who use NSAIDs chronically than for those who are not on chronic NSAIDs. ${ }^{13}$ The main clinical advantage for COX-2 specific inhibitors is the potential decrease in GI events. ${ }^{7-9,13}$ The efficacy for both COX-2 specific inhibitors and nonspecific NSAIDs in reducing pain caused by arthritis is similar, if not equal. ${ }^{7,9,14}$ 
COX-2 specific inhibitors work by inhibiting prostaglandin synthesis by selectively inhibiting the cyclooxygenase-2 enzyme without inhibiting the cyclooxygenase-1 enzyme, unlike nonspecific NSAIDs, which inhibit both COX-1 and COX-2 enzymes. Due to the selectivity of the COX-2 enzyme, this class of medications does not exhibit antiplatelet activity, and a decreased incidence of GI effects has been reported. ${ }^{6,15,16}$ The side-effect profile of COX-2 specific inhibitors has been shown to produce a lower rate of GI events compared to traditional NSAIDs. At 2 to 4 times the indicated dose, celecoxib $400 \mathrm{mg}$ twice daily had resulted in fewer ulcers, both symptomatic ulcers and ulcer complications, compared to NSAIDs. ${ }^{15}$ Results with rofecoxib showed significantly fewer GI events than with nonselective inhibitors, specifically naproxen. ${ }^{16}$

Use of PA, in general, has been shown to result in a significant decrease in pharmacy costs for medications that have lower-cost therapeutic alternatives. There has been a documented drug-cost savings resulting either from use of lower-cost drugs or a decrease in drug utilization as an outcome of PA. ${ }^{17} \mathrm{PA}$ could be a cost-effective method for use of medications that vary greatly in price but not in efficacy. ${ }^{18}$ Previous studies include work that found drug benefit design with PA reduced target drug costs by $49.8 \% .^{19}$

Yet, PA may not be a popular cost-savings method among providers and patients because some find it burdensome. Therefore, it is important to evaluate not only cost savings but also how this intervention and process affects medical and service (e.g., satisfaction) outcomes. Kotzan et al. stated:

The long-term impact of PA programs has not been documented. If the drug programs are devised solely on the basis of economic consideration without regard for medical consequences, then it is possible that more expensive services will replace those expensive drugs removed from the formulary. ${ }^{20}$

In the year 2000, the PA call volume in this MCO showed that the most requested class of medications was the COX-2 specific inhibitors. It accounted for $25 \%$ of the call volume, followed by proton pump inhibitors (PPIs), which accounted for $20 \%$ of the total calls (data not presented).

Historically, in this MCO health plan, PA on COX-2 specific inhibitors was associated with a lower use of COX-2 drugs and appeared to provide cost savings. The prescription drug market share of COX-2 specific inhibitors compared to all NSAID prescriptions in the MCO was $9 \%$. This was lower than the national COX-2 prescription market share of $19 \%$, as reported by IMS Health for July 1999. ${ }^{21}$

The MCO's market share was also significantly lower compared to a $40 \%$ COX-2 market share for an unmanaged benchmark population with no restrictions on COX-2 specific inhibitors, based on information provided by the PBM. The calculated savings based on observed lower utilization attributed to the PA on COX-2 specific inhibitors was $\$ 0.31$ per member per month (PMPM). Operating the PA call center is associated
TABLE 1 COX-2 Monthly Approval Rate Based on Each Criterion for March 2000

\begin{tabular}{l|c|c}
\hline PA Criteria & \% approved & $\begin{array}{c}\text { \# of requests } \\
\text { approved }\end{array}$ \\
\hline Failure of 2 different nonspecific NSAIDs & $73 \%$ & 1,644 \\
\hline Concomitant use of: PPI & $6 \%$ & 135 \\
\hline Oral steroid & $1 \%$ & 23 \\
\hline Anticoagulation or antiplatlet therapy & $2 \%$ & 45 \\
\hline Bisphosphonate & $1 \%$ & 22 \\
\hline Antineoplastic agent & $2 \%$ & 2 \\
\hline History of PUD, GI bleed, hemophilia, & $15 \%$ & 15 \\
\hline Von Willebrand's disease, thrombocytopenia &
\end{tabular}

with many administrative costs. The administrative cost per call in 2000 was determined to be $\$ 0.07$ PMPM for the entire call center. After paying the entire cost of the clinical pharmacy review call center, the PA on COX-2 specific inhibitors alone saved a net $\$ 0.24$ PMPM $(\$ 0.31-\$ 0.07)$. This equates to annual savings of almost $\$ 10$ million for PA on COX-2 specific inhibitors in this $\mathrm{MCO}$ of 3.2 million members with a pharmacy benefit.

This study is a comparison between the utilization and predicted serious GI adverse event occurrence for members on nonspecific NSAIDs versus COX-2 specific inhibitors, to permit determination of the cost-effectiveness of these medications with and without PA. The decision analysis takes into account the changes in utilization with and without PA as well as the cost of treatment for both benefit design options. Treatment cost will include not only drug costs but also the medical and hospital costs to treat adverse events, specifically serious GI events.

\section{Methods}

\section{Data Source}

Prescription drug claims from a health plan with 3.8 million $\mathrm{HMO}$ and PPO members were utilized for this study. At the time of the study, this MCO placed COX-2 inhibitors in the third copay (\$30) tier and required PA. Generic NSAIDs were in the first copay (\$5) tier, and brand-name NSAIDs (both singlesource brand and multiple-source brand) were in the second copay (\$15) tier. The review criteria used for this PA program included (a) failure or intolerance of 2 different NSAIDs or (b) evidence of adverse GI risk factors, such as concomitant use of steroids, proton pump inhibitors (PPIs), or prescriptionstrength histamine blockers, anticoagulant or antiplatelet therapy, bisphosphonates, or antineoplastic agents. Nondrug-related risk factors were also assessed, including either a history of or current GI bleed or ulcer. At the time of the study, no electronic step-therapy edit was in place; therefore, all criteria were assessed by physician-reported patient use of these medications and patient risk factors. Along with physician-reported patient use, drug claims history was assessed at the time of each request 
to look for prior use of medications. Forty-five percent of requests were approved, which means that the member met both prior NSAID use and the risk-factor criteria. Of those denied, $66 \%$ failed to meet the NSAID prior-use criteria, 24\% failed to meet the risk factor criteria, and the remaining 10\% failed both criteria.

Table 1 shows the percent of claims approved based on each criteria level. The first criterion was failure of 2 different nonspecific NSAIDs. If the physician or the claims data verified use of 2 NSAIDs, a COX-2 specific inhibitor was automatically approved without any further check of criteria.

\section{Literature Evaluation}

A literature search was performed to determine the serious GI side-effect probabilities for each treatment option. The MEDLINE database was searched for clinical trials and review articles relating to nonspecific NSAIDs, COX-2 specific inhibitors, arthritis, and GI adverse effects. The search included human, adult studies from 1995 to 2001. Data were also gathered from American Hospital Formulary Source (AHFS) Drug Information, the package inserts of nonspecific NSAIDs and COX-2 specific inhibitors, and FDA transcripts from advisory committee meetings. Data compiled from the above literature sources provided the incidence for serious GI adverse events and/or reduction in incidence of serious GI events when a gastroprotective agent, specifically a PPI, is added to NSAID therapy. The probabilities were determined based on published literature. The sample size in this study was so large that we could assume that our population would experience side effects in both incidence and severity similar to those in the published reports.

Based on the published literature, several assumptions were made. The side-effect frequency was categorized by drug class and not by individual agent. These categories included COX-2 only, nonspecific NSAID only, COX-2 specific inhibitor plus a PPI, nonspecific NSAID plus a PPI, and no drug therapy. The probability of chronic nonspecific NSAID users developing upper GI ulcers, bleeding, and/or perforation within a 1-year period was assumed to be $2 \%$ to $4 \%{ }^{6}$ This decision analysis used a mean probability of $3 \%$. The background probability of the general population experiencing a serious GI event is $0.4 \% .^{22}$ This same incidence was used to estimate the probability of the COX-2 specific inhibitor population experiencing a serious GI side effect.

Adding a PPI for gastroprotection to either a nonspecific NSAID or COX-2 specific inhibitor reduces the risk of a serious GI side effect by approximately 50\%. 10,13,23 Therefore, we assumed the GI protective effect of a PPI combined with COX2 specific inhibitor would be at least equivalent to a PPI combined with a nonspecific NSAID. Assuming an average 50\% risk reduction with the addition of a PPI, the probability of a GI event with a combination of PPI and nonspecific NSAID therapy becomes $1.5 \%$. The probability of a serious GI event for a PPI combined with COX-2 specific inhibitor is $0.2 \%$ based on the same risk reduction. A sensitivity analysis was performed for each probability to account for any assumptions made.

\section{Cost-effectiveness Analysis}

A cost-effectiveness analysis (CEA), using a decision tree, was used to evaluate the option of removing the PA on COX-2 specific agents. The tree was divided into 2 main branches: maintaining PA with the current criteria at the time of the study or removing PA. The subdivisions on each branch vary, including combinations of therapy and probability of serious GI side effects. The distribution of use of each medication combination is based on actual health plan data in pharmacy claims. The probabilities of a serious GI event came from published literature. Only those events defined as serious were shown in the model. For this CEA, we define all costs of therapy, including drug cost and cost of treatment for serious GI event, by cost per success. Success was defined as no serious adverse drug event.

All possible treatment combinations were analyzed, using the health plan pharmacy claims database, including COX-2, COX-2 with PPI, NSAID only, NSAID with PPI, and no prescription drug treatment. The cost and probability of each success and each adverse event were compared within the 2 different benefit models, which include the cost associated with maintaining PA on COX-2 specific inhibitors using the current PA criteria or eliminating the criteria-based PA requirement completely.

The cost in the model represents the actual cost of the claim to the health plan. Actual cost was calculated using the acquisition cost of the medication and the medical costs associated with a serious GI side effect. This cost is reported as annual cost and represents net health plan cost (i.e., cost after subtraction of member copay). No drug manufacturer rebates were factored since none were in place for COX-2 specific inhibitors at the time of the study. The cost of a serious side effect can include other required medications, physician visits, hospital costs, etc.

The cost of a serious GI side effect was classified as any cost associated with a medical claim correlating to a primary or secondary ICD-9 diagnosis code beginning with 531 (gastric or stomach ulcer). The data included all members with those ICD-9 codes, not just members receiving NSAIDs. ICD-9 codes include gastric ulcers with or without perforation or hemorrhage. These costs are an average of medical claims associated with these adverse events for a 1-month snapshot. The actual average cost to the health plan for a serious GI adverse event from medical claims data in November 2000 was $\$ 1,500$ per event. There were just fewer than 1,000 medical claims per ICD-9 code for about 200 individuals, with total plan costs exceeding $\$ 300,000$. The data were limited to just 1 month to minimize the probability that there was not more than 1 event per person. The "event" could include, but was not limited to, hospitalizations.

Previous studies have quoted treatment costs per event as high as $\$ 15,000 . .^{24}$ To account for the wide variability of the health plan's actual cost of a serious GI event compared to reports in 


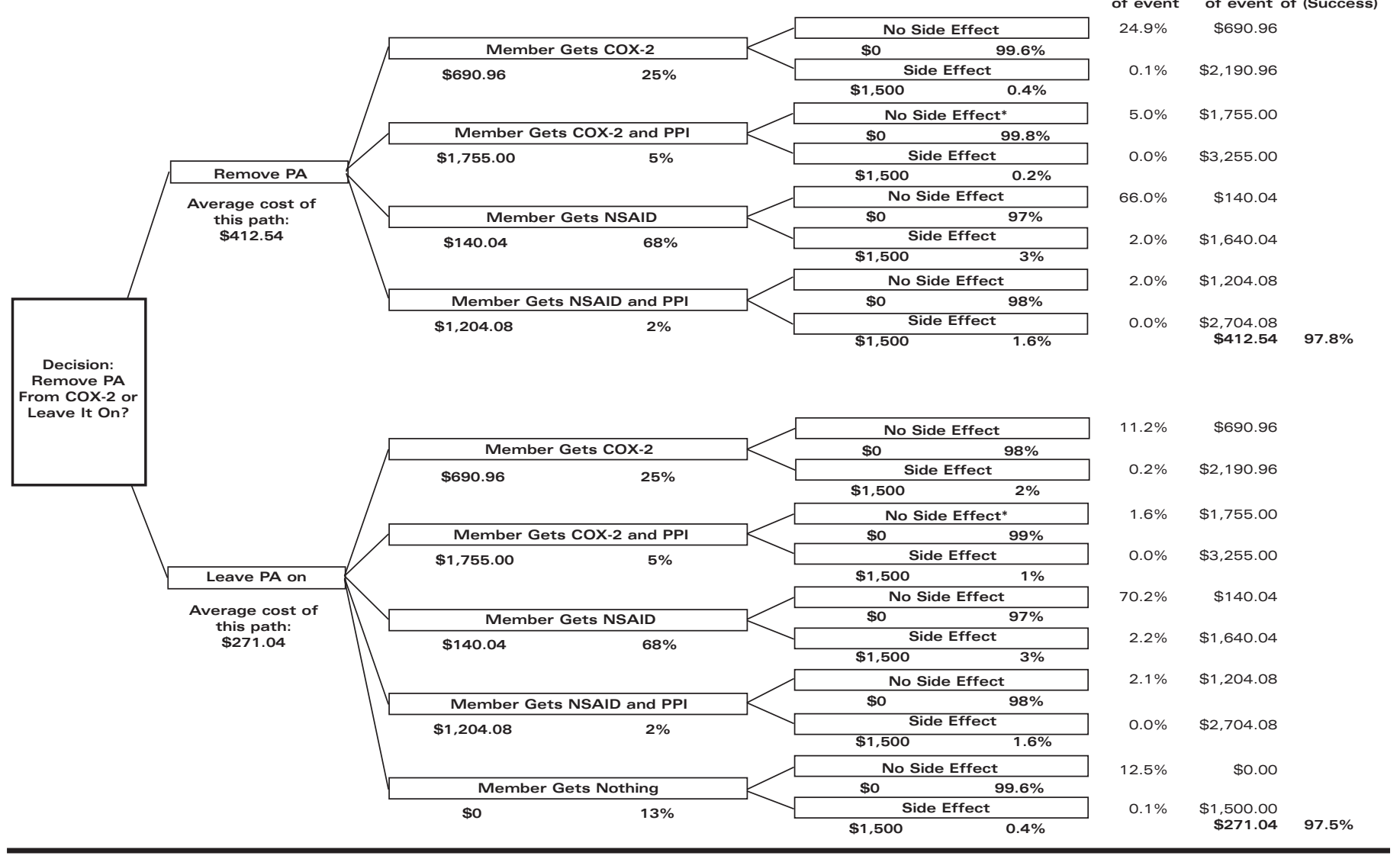

published literature, a sensitivity and threshold analysis was performed. The sensitivity analysis showed that no change in study results was evident until the cost of a serious GI event was greater than $\$ 100,000$. The costs in the model are based on 1 year of treatment and assume 100\% compliance (12 fills per year) and not more that 1 serious event per member per year.

Table 2 lists each cost included in our CEA decision-tree model, including the cost of a serious GI event and cost of the drug for a 1-year time period. Figure 1 shows the decision tree used in the cost-effectiveness model. The tree is divided into 2 main branches. One branch includes the path when the PA is left on COX-2 specific inhibitors. This path shows percentages of members on each treatment branch and the percent probability of members in each path experiencing a serious GI event. The other branch depicts potential utilization when the PA is removed and how this shift in utilization affects the overall rate of serious GI events. Using the costs from Table 2, the cost for each branch was calculated, and, ultimately, the cost of each of the 2 major paths. It could then be determined whether it was cost effective to leave the PA on COX-2 specific inhibitors, taking into account both drug utilization and rate of serious GI side effects.

\section{Population}

The population of the cost-effectiveness model consisted of 96,154 members, or nearly 3\% of the plan's total membership with a drug benefit. This population was defined as all NSAID users and potential NSAID users. Potential NSAID users were defined as those members who tried to get a COX-2 specific inhibitor but whose claim was rejected at the pharmacy.

Figure 2 shows the percent of members who originally met criteria for PA and were approved for the drug, members who originally did not meet criteria and were rejected, and members who did not attempt to obtain a PA approval and alternatively used a nonspecific NSAID. The members who originally did not meet criteria were then analyzed the month following their original rejection to determine if they were approved on a future PA attempt, alternatively used a nonspecific NSAID, or filled no drug in the NSAID class. Specifically, each member included in the study population had received a nonspecific NSAID, received a COX-2 specific inhibitor, or tried to obtain a PA on a COX-2 specific inhibitor during January 2001. The percentage of members in each category was $71 \%, 8 \%$, and $21 \%$, respectively. Of $21 \%$ of members who tried to get a COX-2 but were denied by the crite- 


\begin{tabular}{lc}
\hline \multicolumn{1}{l}{ TABLE 2} & Costs* in the CEA Model \\
\hline Serious GI Event & $\$ 1,500 /$ event \\
\hline COX-2 treatment & $\$ 691 / 12$ monthly claims \\
\hline NSAID treatment & $\$ 140 / 12$ monthly claims \\
\hline COX-2 \& PPI treatment & $\$ 1,755 / 12$ monthly claims \\
\hline NSAID \& PPI treatment & $\$ 1,204 / 12$ monthly claims \\
\hline $\begin{array}{l}\text { Net plan costs after subtraction of member cost-share for claims with dates of } \\
\text { service from November 2000 to February }\end{array}$ & 2001. \\
\hline
\end{tabular}

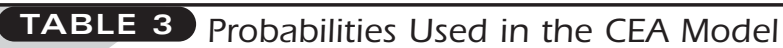

\begin{tabular}{l|c|c|c}
\hline Medication & $\begin{array}{c}\text { Probability of } \\
\text { Serious GI Event, } \\
\text { From Literature }\end{array}$ & $\begin{array}{c}\text { Probability } \\
\text { Used in the PA } \\
\text { Arm of Model }\end{array}$ & $\begin{array}{c}\text { Probability } \\
\text { Used in the } \\
\text { No-PA Arm } \\
\text { of Model }\end{array}$ \\
\hline COX-2 & $2 \%^{6}$ & $2 \%$ & $0.4 \%$ \\
\hline Nonspecific NSAID & $2 \%$ to $4 \%^{6}$ & $3 \%$ & $3 \%$ \\
\hline COX-2 and PPI & $\begin{array}{c}30 \% \text { to } 75 \% \text { decrease } \\
\text { risk of NSAID alone } \\
10,13,23\end{array}$ & $1 \%$ & $0.2 \%$ \\
NSAID and PPI & $\begin{array}{c}30 \% \text { to } 75 \% \text { decrease } \\
\text { risk of NSAID alone } \\
10,13,23\end{array}$ & $1.5 \%$ & $1.5 \%$ \\
\hline No NSAID & $0.4 \%{ }^{6}$ & $0.4 \%$ & $0.4 \%$ \\
\hline
\end{tabular}

\section{FIGURE 2 Population of the CEA Model}

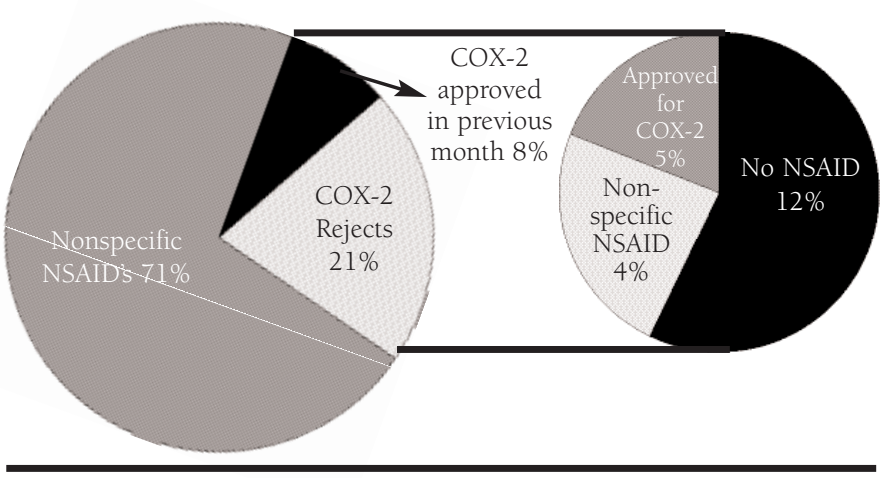

ria, $12 \%$ received no NSAID, $5 \%$ were later approved for a COX2 , and $4 \%$ filled a nonspecific NSAID instead.

Actual health plan data were used to determine utilization and prescribing patterns of nonspecific NSAIDs, COX-2 specific inhibitors, and PPIs within the current benefit design at the time of the study. The alternate benefit design based on removal of prior authorization assumes no restrictions placed on prescriptions for COX-2 specific inhibitors. Therefore, utilization is estimated based on both current utilization of members with PA
(15\% of NSAID market share), rejection rate of COX-2 specific inhibitors (29\% of population who tried to obtain a COX-2 specific inhibitor), and reported benchmarks of COX-2 utilization (IMS data of $19 \%$ market share ${ }^{21}$ ).

The probabilities of having a serious GI event, obtained from the published literature (Table 3), were then placed in the decision-tree model. It was assumed that the published probabilities applied to the general population without restrictions, and, therefore, these numbers related to the branch without a PA. To determine the GI adverse events within the PA branch, it was assumed that those who receive a COX-2 specific inhibitor within the PA process are at a higher risk because they must meet risk criteria to receive approval for the medication. For this high-risk population, it was estimated that the population with a PA already in place for a COX-2 specific inhibitor is 5 times more likely to have a side effect. This was based on the $0.4 \%$ risk reported in the general background population and assumed a minimal 2\% incidence of serious GI adverse effects from members taking chronic NSAIDs.

\section{Outcomes Measured}

The primary outcome measure was the average cost per successful treatment. A successful treatment was defined as no serious GI event. This outcome was measured for both the prior authorization benefit and the proposed benefit without PA for COX-2 specific inhibitors. The costs associated with PA and without PA were calculated to determine if changes in utilization when PA is removed would have an impact on the number of successes and therefore decrease the cost of treating serious GI events enough to justify PA removal.

\section{Results}

In the 3-tier copay design in this MCO health plan that required PA for COX-2 specific inhibitors, the average annual cost across all therapeutic possibilities for the study population was $\$ 271.04$ per patient. This number is an average of all study treatment possibilities, including the cost of treating serious Gl adverse effects. If the PA on COX-2 specific inhibitors was removed, and $100 \%$ of the study population who attempted to fill a COX-2 specific inhibitor received a COX-2 specific agent (i.e., all prescriptions presented to the pharmacy were dispensed), then the annual average cost of therapy would be $\$ 412.54$ per patient, assuming 12 fills per year.

CEA compared the cost per success for COX-2 specific inhibitors with PA versus the cost per success without PA. The cost was $\$ 277.99$ per success for PA and $\$ 421.82$ per success without PA. Each additional 1\% increase in the probability of having no serious GI side effect would cost the health plan an additional $\$ 47,167$ per member per year for each member on a COX-2 specific inhibitor if the PA was removed. This equates to more than $\$ 13.6$ million a year for the entire study population $(\mathrm{N}=96,154)$, assuming that all prescription claims for COX-2 specific inhibitors are no longer rejected and members are 100\% compliant (12 fills 
per year) with their treatment.

The national risk of serious GI event over the entire population is $0.4 \% \cdot{ }^{4,5}$ When the national population is compared to the study population, $13 \%$ of the study population exhibits some level of risk for GI complications. This is evident by the percentage of members who met the PA criteria and are therefore considered the higher-risk population. This higher-risk population in the study group could potentially sway results to make removal of PA seem more beneficial. In spite of this possible bias to PA removal, maintaining PA still proved to be a more favorable option.

To account for all assumptions in the model, a sensitivity analysis was performed on all probabilities and costs associated with serious adverse events. The predicted outcomes were not significantly changed even when probabilities and cost were adjusted for both sensitivity and threshold analysis. Removing the PA for the 3-tier benefit design was always a more costly decision (Figure 3).

There has been continued controversy around the exact GI risk reduction associated with COX-2 specific inhibitors versus nonspecific NSAIDs. The CLASS (Celecoxib Long-term Arthritis Safety Study) ${ }^{15}$ clinical trial and VIGOR (Vioxx Gastrointestinal Outcomes Research $)^{16}$ study and other studies have brought up many questions around the potential risks and benefits of COX-2 specific inhibitors and the populations in which COX-2 specific inhibitors are most appropriate. These studies were published after the completion of this CEA but now provide a wonderful opportunity to reevaluate COX-2 specific inhibitor use and specifically evaluate actual rates of serious GI events that occurred within this health plan. A study by Bull et al. showed that prescribing patterns have begun to shift to higherrisk patients receiving COX-2 specific inhibitors and lower-risk patients receiving nonspecific NSAIDs based on a COX-2 risk score. ${ }^{25}$ As prescribing patterns and patient demand for these products begin to decrease and use is shifted to high-risk populations, the need and value of PA decreases.

The value of PA will continue to be discussed and debated. An analysis of the experience of a PA program in a Medicaid HMO showed that $95 \%$ of PA requests were approved, thereby questioning the value of PA. ${ }^{26}$ Nevertheless, Medicaid plans will likely maintain PA programs. A recent study from Hamer et al. suggested that the addition of PA for gabapentin could be used to decrease the prescribing of off-label uses of the drug. ${ }^{27}$ This fuels the debate on the use of PA to both reduce costs and achieve appropriate utilization.

Another factor to seriously consider when determining the value of PA is the effect on patient and provider satisfaction. The published studies that have examined the effects of PA programs have not measured either service outcomes (satisfaction with care) or health-related quality of life. ${ }^{28}$ Although our study showed a definite cost savings associated with PA on this particular class of drugs, the value may decrease when considering member retention, satisfaction, and the burden of use associat-

\section{FIGURE 3 Sensitivity Analysis for All Probabilities}
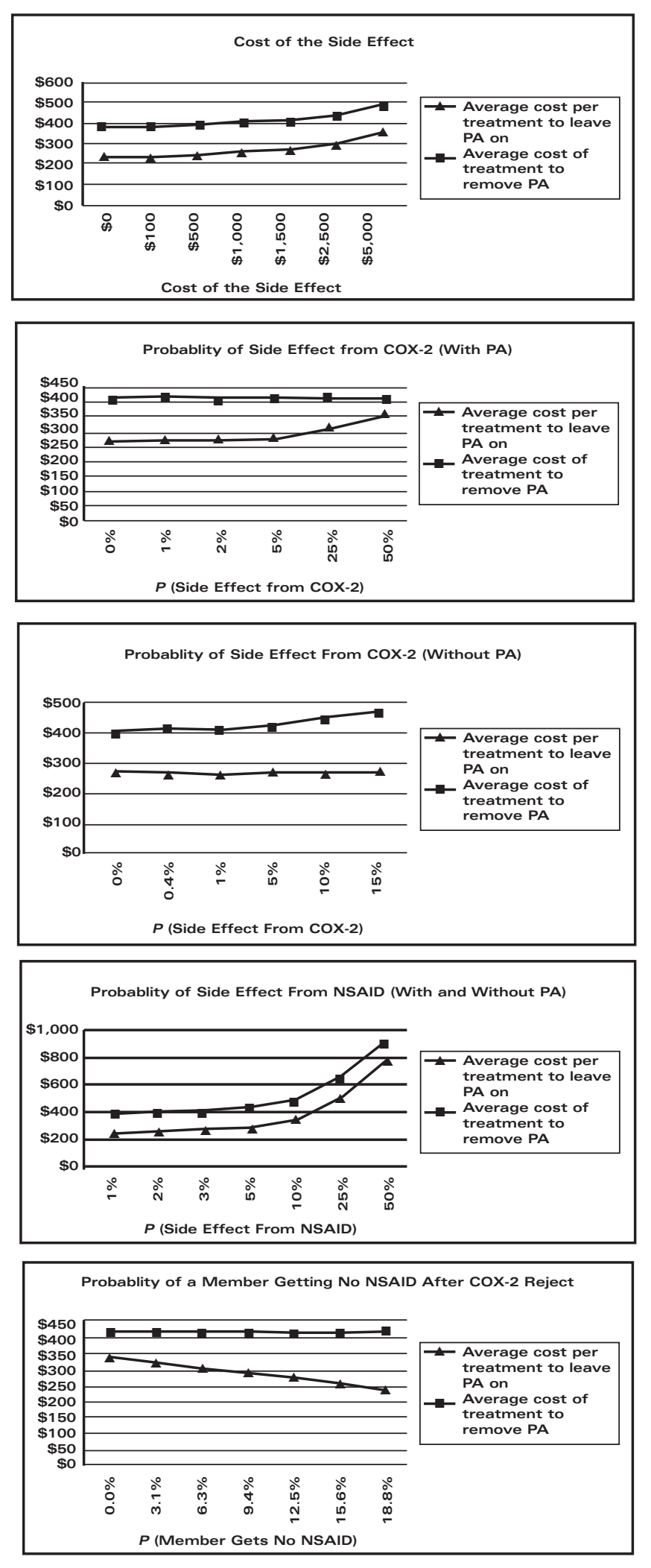
ed with the PA process.

Based on the results of our study, PA remains in place in this health plan for COX-2 specific inhibitors in the 3-tier and traditional drug benefit designs, but the PA program was removed due to concerns regarding member and provider satisfaction in the redesign of this health plan's 4-tier drug benefit. When health plans consider a benefit design without PA, it is important that pharmacy managers and health plan actuaries take into account the predicted increased utilization of medications previously requiring PA and price the new benefit accordingly.

\section{Limitations}

There are several limitations of this study. While actual health plan data were used to measure utilization, cost of treatment, and population distribution, the probabilities of serious GI events were obtained from the published literature and not from actual events observed in our health plan. Further studies will be necessary to confirm the estimated rate $(0.4 \%$ to $3 \%$, depending on the drug) of adverse drug events in the health plan. Second, we assumed 100\% compliance with drug treatment, since one objective of the study was to estimate the maximum cost to the health plan of discontinuation of the PA requirement. Cost of drug treatment could be overstated in this study if the population was not 100\% compliant with the drug regimens.

This CEA focused solely on the PA process, and the influence of tiered copayments was not evaluated. It included only serious GI events; GI discomfort or any moderate or mild GI symptoms were not included. This study examined classes of drugs and did not evaluate specific drugs within the classes. Pharmacy claims data were used to calculate an average cost per therapeutic class, averaging the variation in cost by specific drug and dose. This method has limitations as well as potential value given the evolving body of knowledge regarding the relative differences among specific COX-2 drugs and NSAIDs in the incidence of adverse cardiac and renal effects, outcomes that were also not examined in this PA CEA.

\section{Conclusions}

Maintaining PA on COX-2 specific inhibitors can be a cost-effective tool to assure that target (higher-risk) members receive this treatment while those who are at low risk for adverse GI events use the nonspecific NSAIDs as first-line therapy. More than 15,000 members during the study period either did not meet risk criteria or their physicians did not try to obtain a PA. These members therefore received either a nonspecific NSAID or no NSAID therapy. Due to the variation of prescribing patterns for COX-2 specific inhibitors, significant DTC advertising, and the cost of COX-2 specific inhibitors compared to their equally efficacious NSAID alternatives, PA can be an effective tool to control costs in either a 3-tier copay design or a traditional drug benefit design. This CEA showed that the greater medication expense of COX-2 specific inhibitors versus nonspecific
NSAIDs cannot be outweighed by the side-effect profiles of these 2 classes of medications. The risk factors for GI events are considered in the COX-2 inhibitor PA criteria, and, therefore, PA for COX-2 specific inhibitors remains a cost-effective managed care intervention when evaluating overall medical and pharmacy costs.

\section{DISCLOSURES}

Funding for this research was provided by Humana Inc. Corporate Pharmacy Management and was obtained by authors Jane Stacy, Elizabeth Shaw, Michele D. Arledge, and Donna Howell-Smith. All authors are employed by Humana Inc.; they reported no biases or conflicts of interest in the preparation of this manuscript. Stacy served as principal author of the study. Study concept and design were contributed by Stacy, Howell-Smith, and Shaw. Analysis and interpretation of data were contributed by all authors. Drafting of the manuscript was primarily the work of Stacy and its critical revision was the work of Arledge. Statistical expertise was contributed by Shaw and administrative, technical, and/or material support was provided by Humana Inc.

\section{REFERENCES}

1. Vogel RJ, Ramchandran S, Zachry WM, et al. A 3-stage model for assessing the probable economic effects of direct-to-consumer advertising of pharmaceuticals. Clin Ther. 2003;25(1):309-29.

2. Young D. Studies show drug ads influence prescription decisions, drug costs. Am J Health-Syst Pharm. 2002;59(1):14-16.

3. Momani A, Odedina F, Rosenbluth S, Modhaven S. Drug-management strategies: Consumers' Perspectives. J Managed Care Pharm. 2000;6(2):122-28.

4. Celebrex prescribing information. Chicago, IL: G.D. Searle, LLC; 2002.

5. Vioxx prescribing information. Whitehouse Station, NJ: Merck; 2002.

6. McEvoy GK., ed. AHFS Drug Information 2001. Bethesda, MD: American Society of Health-System Pharmacists; 2000.

7. Deeks JJ, Smith LA, Bradley MD. Efficacy, tolerability, and upper gastrointestinal safety of celecoxib for treatment of osteoarthritis and rheumatoid arthritis: systematic review of randomised controlled trials. BMJ. 2002;325 (7365):619.

8. Simon LS, Smolen JS, Abramson SB, et al. Controversies in COX-2 selective inhibition. J Rheumatol. 2002;29(7):1501-10.

9. Ruffalo R, Jackson R, Ofman J. The impact of NSAID selection on gastrointestinal injury and risk for cardiovascular events: identifying and treating patients at risk. Pharm Ther. 2002;27(11):570-76.

10. Chancellor JV, Hunsche E, de Cruz E, Sarasin FP. Economic evaluation of celecoxib, a new cyclo-oxygenase 2 specific inhibitor, in Switzerland. Pharmacoeconomics. 2001;19(suppl 1):

59-75.

11. Hernandez-Diaz, S, Rodriguez LA. Association between nonsteroidal antiinflammatory drugs and upper gastrointestinal tract bleeding/perforation; an overview of epidemiologic studies published in the 1990s. Arch Intern Med. 2000;160(14):2093-99.

12. Goldstein JL. Who needs prophylaxis of nonsteroidal anti-inflammatory drug-induced ulcers and what is optimal prophylaxis? Eur J Gastorenterol Hepatol. 2000;12(suppl 1):S11-S15.

13. La Corte R, Caselli M, Castellino G, et al. Prophylaxis and treatment of NSAID-induced gastroduodenal disorders. Drug Safety. 1999;20(6):527-43.

14. Cannon GW, Breedveld FC. Efficacy of cyclooxygenase-2-specific inhibitors. Am J Med. 2001;110 (suppl 3A):6S-12S

15. Silverstein FE, Faich G, Goldstein JL, et al. Gastrointestinal toxicity with celecoxib vs. nonsteroidal anti-inflammatory drug for osteoarthritis and rheumatoid arthritis: the CLASS study: a randomized controlled trial. Celecoxib Long-term Arthritis Safety Study. JAMA. 2000;284(10):1247-55. 
16. Bombardier C, Laine L, Reicin A, et al. VIGOR Study Group.Comparison of upper gastrointestinal toxicity of rofecoxib and naproxen in patients with rheumatoid arthritis. N Engl J Med. 2000;343(21):1520-28.

17. MacKinnon N, Kumar R. Prior authorization programs: A critical review of the literature. J Managed Care Pharm. 2001;7(4):297-302.

18. Smalley WE, Griffin MR, Fought RL, Sullivan L, Ray WA. Effect of a priorauthorization requirement on the use of nonsteroidal anti-inflammatory drugs by Medicaid patients. New Engl J Med. 1995;332(24):1612-17.

19. Tucker G, Moore A, Avant D, Monteiro M. A cost analysis of four benefit strategies for managing a Cox II inhibitor. J Managed Care Pharm. 2001;7(3):224-27.

20. Kotzan JA, Perri M, Martin BC. Assessment of Medicaid prior-approval policies on prescription expenditures: market-share analysis of Medicaid and cash prescriptions. J Managed Care Pharm. 1996;2(6):651-56

21. IMS Health Data. July 1999. Available at: http://www.imshealth.com. Accessed September 21, 1999

22. FDA Center for Drug Evaluation and Research. Arthritis Advisory Committee meeting transcript. Feb.7, 2001. Available at:

http://www.fda.gov/ohrms/dockets/ac/transcripts/3677tl.rtf. Accessed April 2001.
23. Burke TA, Zabinski RA, Pettitt D, Maniadakis N, Maurath CJ, Goldstein JL. A framework for evaluating the clinical consequences of initial therapy with NSAIDs, NSAIDs plus gastroprotective agents, or celecoxib in the treatment of arthritis. Pharmacoeconomics. 2001;19(suppl 1):33-47.

24. Wolfe, MM, Lichtenstein, DR, Singh G. Medical progress: gastrointestinal toxicity of nonsteroidal anti-inflammatory drugs. New Engl J Med. 1999;340(24):1888-99.

25. Bull SA, Conell C, Campen DH. Relationship of clinical factors to the use of COX-2 selective NSAIDs within an arthritis population in a large HMO. J Managed Care Pharm. 2002;8(4):252-58.

26. LaPensee KT. Analysis of a prescription drug prior authorization program in a Medicaid health maintenance organization. J Managed Care Pharm. 2003;9(1):36-44.

27. Hamer AM, Haxby DG, McFarland BH, Ketchum K. Gabapentin use in a managed Medicaid population. J Managed Care Pharm. 2002;8(4):266-71.

28. Curtiss FR. Managing care via prior authorization (PA) programs? J Managed Care Pharm. 2002;8(4):296. 